\title{
Three-Prey One-Predator Continuous Time Nonlinear System Model
}

\author{
Jingyuan Zhang $(\mathbb{D})$ and Yan Yang $(1 D$ \\ Business School, Hunan University, Changsha 410082, Hunan, China \\ Correspondence should be addressed to Yan Yang; yangyan@hnu.edu.cn
}

Received 27 August 2020; Revised 19 September 2020; Accepted 30 September 2020; Published 10 October 2020

Academic Editor: Chun-Biao Li

Copyright (c) 2020 Jingyuan Zhang and Yan Yang. This is an open access article distributed under the Creative Commons Attribution License, which permits unrestricted use, distribution, and reproduction in any medium, provided the original work is properly cited.

\begin{abstract}
In this paper, we propose a new multiple-prey one-predator continuous time nonlinear system model, in which the number of teams of preys is equal to 3; namely, a continuous time three-prey one-predator model is put forward and studied. The fourthorder differential equation is established, in which the prey teams help each other. The equilibrium points and stability are analyzed. When not considering preys help each other, we study the global stability and persistence of the model without help terms. The simulation results of system solutions with help terms corresponding to locally asymptotically stable equilibrium points and without help terms corresponding to globally asymptotically stable equilibrium points are given.
\end{abstract}

\section{Introduction}

Nonlinear systems exist in many ways with various research directions, such as chaotic circuit [1-8], neural network [9-15], and image encryption [16-18]. Prey-predator systems also have nonlinear characteristics. In 1965, Holling proposed the functional responses of three different species to simulate predation. These functional responses describe how predators transform the captured prey into their own growth. According to the research, the prey-predator model can be divided into four categories: (i) the first category is the single-prey single-predator model; (ii) the second category is the single-prey multipredator model; (iii) the third category is the multiprey multipredator model; and (iv) the fourth category is the multiprey single-predator model. There are many research studies on the first type of the model. For example, Rosenzweig and MacArthur [19] proposed the Rosenzweig-MacArthur model (R-M model) or the predator-prey model with Holling type II functional response and gave graphical presentation and stability conditions of predator-prey interactions. Hsu and Huang [20] studied global stability of the R-M model. In [21], the conditions of internal equilibrium were given and the stability of internal equilibrium was analyzed. It also discussed certain critical situations, some of which cannot occur in the usual model.
Wang and Jing [22] studied the global stability and the existence of limit cycles of a predator-prey system. The existence of at least two limit cycles was proved by qualitative analysis and Poincare-Bendixson theory. Zhang and Hou [23] investigated a nonautonomous ratio-dependent predator-prey model with exploited terms. By using the coincidence degree theory, it was proved that there are at least four positive periodic solutions. For the second category, Llibre and Xiao [24] studied the competitive exclusion of two predators and one prey. In the absence of predators, the growth rate of the prey was logistic or linear. The boundedness of the solution was proved by studying the stability of the equilibrium point of the differential equation at infinity. Through the theoretical analysis of the equations, the necessary and sufficient conditions for the existence of the exclusion principle were obtained, and the global dynamic behaviors of the three species in the first octaves were given. Wang et al. [25] studied a class of $n$-dimensional consumerresource systems, in which a group of consumers compete for the same resource, and each consumer is mutually beneficial with the resource. Wang and $\mathrm{Wu}$ [26] considered the global dynamics of an $n$-dimensional Lotka-Volterra system in which $(n-1)$ predator species compete for a single prey species. By using the theory of dynamic systems, the global dynamic behaviors of $n$ species in the first octant were 
given, and sufficient and necessary conditions for the existence of the competitive exclusion principle were proved, thus extending Volterra's principle and the work of Llibre and Xiao [24]. For the third category, Elettreby and ElMetwally [27] gave models in which two teams of predators interact with two teams of preys. The teams in each group (predators or preys) help each other. Three different versions of the multiteam predator-prey model were proposed. In addition, the equilibrium solutions, the conditions of their local asymptotic stability, and the global stability of the solution of one of the models were studied. For the fourth category model, Elettreby [28] proposed a two-prey onepredator model, in which the prey teams help each other. Its local stability was studied. In the absence of predators, there was no help between the prey teams. The global stability and persistence of the model without help were studied. However, for the continuous time multiple-prey one-predator model, so far, only the above two-prey one-predator model has been proposed. No continuous time multiple-prey onepredator model in which the number of teams of preys is greater than 2 has been proposed. But in practice, in many cases, the number of teams of preys is greater than 2 . Therefore, in this paper, we propose a new multiple-prey one-predator model, in which the number of teams of preys is equal to 3; namely, a continuous time three-prey onepredator model is put forward and studied. The fourth-order differential equation is established. The equilibrium points and stability are analyzed.

\section{The Model}

In this section, we propose a system consisting of three teams of preys with densities $x, y$, and $z$, respectively, interacting with one team of predator with densities $w$. The assumptions of this model are as follows:

(1) In the absence of any predation, each team of preys grows logistically, that is, $a x(1-x), b y(1-y)$, and $c z(1-z)$.

(2) The effect of the predation is to reduce the prey growth rate by a term proportional to the prey and predator populations, that is, the $-x w,-y w$, and $-z w$ terms.

(3) The teams of preys help each other against the predator, that is, a $x y z w$ term exists.

(4) In the absence of any prey for sustenance, the predator's death rate results in inverse decay, that is, the term $-\mathrm{d} z^{2}$.

(5) The prey's contribution to the predator growth rate is $\mathrm{e} x w$, fyw, and $\mathrm{g} z w$, which is proportional to the available prey as well as the size of the predator population.
Using the above assumptions, the following model is proposed:

$$
\left\{\begin{array}{l}
\frac{\mathrm{d} x}{\mathrm{~d} t}=a x(1-x)-x w+x y z w, \\
\frac{\mathrm{d} y}{\mathrm{~d} t}=b y(1-y)-y w+x y z w, \\
\frac{\mathrm{d} z}{\mathrm{~d} t}=c z(1-z)-z w+x y z w, \\
\frac{\mathrm{d} w}{\mathrm{~d} t}=-\mathrm{d} w^{2}+e x w+f y w+g z w,
\end{array}\right.
$$

where the coefficients $a, b, c, d, e, f$, and $g$ are positive constants and the initial values $x(0), y(0), z(0)$, and $w(0)$ are all greater than zero. It is clear that the three teams of preys help each other, e.g., in foraging and in early warning against predation. Note that this help occurs only in the presence of predator. This is presented by the term $x y z w$ in the prey equations.

\section{The Analysis of the Model}

First, let us find the equilibrium point of system (1). In order to find the equilibrium points of equation (1), equation (1) can be set to zero as follows:

$$
\left\{\begin{array}{l}
a x(1-x)-x w+x y z w=0 \\
b y(1-y)-y w+x y z w=0 \\
c z(1-z)-z w+x y z w=0 \\
-d w^{2}+e x w+f y w+g z w=0 .
\end{array}\right.
$$

Solving equation (2), we can obtain the equilibrium point $E(x, y, z, w)$ as follows: $E_{0}(0,0,0,0), E_{1}(1,0,0,0), E_{2}$ $(0,1,0,0), E_{3}(0,0,1,0), E_{4}(0,1,1,0), E_{5}(1,0,1,0), E_{6}(1,1$, $0,0), E_{7}(1,1,1,0), E_{8}(0,0,(d c /(d c+g)),(g c /(d c+g)))$, $E 9(0,(d b /(d b+f)), 0,(f b /(d b+f))), \quad E_{10}((d a / d a+e)$, $0,0,(e a / d a+e)), \quad E_{11}(0,(b c d-c g+b g / b c d+c f+b g)$, $(b c d+c f-b f / b c d+c f+b g), \quad(b c f+b c g / b c d+c f+b g))$, $E_{12} \quad((a c d-c g+a g / a c d+c e+a g), 0,(a c d+c e-a e l a c d+$ $c e+a g), \quad(a c e+a c g / a c d+c e+a g)), \quad E_{13}((a b d-b f+$ $a f / a b d+b e+a f), \quad(a b d+b e-a e / a b d+b e+a f), 0$, $(a b e+a b f / a b d+b e+a f))$, and $E_{14}(1,1,1,(e+f+g / d))$.

Proposition 1. Local stability analysis shows that system (1) has eight unstable equilibrium solutions $E_{0}, E_{1}, E_{2}, E_{3}, E_{4}, E_{5}$, $E_{6}$, and $E_{7}$.

Proof. The Jacobian matrix of system (1) is given by the following:

$$
J=\left[\begin{array}{cccc}
a(1-2 x)-w(1-y z) & x z w & x y w & -x(1-y z) \\
y z w & b(1-2 y)-w(1-x z) & x y w & -y(1-x z) \\
y z w & x z w & c(1-2 z)-w(1-x y) & -z(1-x y) \\
e w & f w & g w & -2 d w+e x+f y+g z
\end{array}\right]
$$


The characteristic equation is as follows:

$$
|J-\lambda E|=\left[\begin{array}{cccc}
a(1-2 x)-w(1-y z)-\lambda & x z w & x y w & -x(1-y z) \\
y z w & b(1-2 y)-w(1-x z)-\lambda & x y w & -y(1-x z) \\
y z w & x z w & c(1-2 z)-w(1-x y)-\lambda & -z(1-x y) \\
e w & f w & g w & -2 d w+e x+f y+g z-\lambda
\end{array}\right]=0 .
$$

By substituting the point $E_{0}(0,0,0,0)$ in equation (4), we get $\lambda=0, a, b$, and $c$, which has three positive eigenvalues. So, it is an unstable equilibrium point.

Similarly, by substituting the point $E_{1}(1,0,0,0)$ in equation (4), we get $\lambda=-a, b, c$, and $e$, which has three positive eigenvalues. So, it is an unstable equilibrium point.

Similarly, by substituting the point $E_{2}(0,1,0,0)$ in equation (4), we get $\lambda=a,-b, c$, and $e$, which has three positive eigenvalues. So, it is an unstable equilibrium point.

Similarly, by substituting the point $E_{3}(0,0,1,0)$ in equation (4), we get $\lambda=a, b,-c$, and $e$, which has three positive eigenvalues. So, it is an unstable equilibrium point.

Similarly, by substituting the point $E_{4}(0,1,1,0)$ in equation (4), we get $\lambda=a,-b,-c$, and $f+g$, which has two positive eigenvalues. So, it is an unstable equilibrium point.

Similarly, by substituting the point $E_{5}(1,0,1,0)$ in equation (4), we get $\lambda=-a, b,-c$, and $e+g$, which has two positive eigenvalues. So, it is an unstable equilibrium point.

Similarly, by substituting the point $E_{6}(1,1,0,0)$ in equation (4), we get $\lambda=-a,-b, c$, and $e+f$, which has two positive eigenvalues. So, it is an unstable equilibrium point.

Similarly, by substituting the point $E_{7}(1,1,1,0)$ in equation (4), we get $\lambda=-a,-b,-c$, and $e+f+g$, which has one positive eigenvalue. So, it is an unstable equilibrium point.

So, all of them are unstable equilibrium points.

By substituting the point $E_{8}(0,0,(d c /(d c+g))$, $(g c /(d c+g)))$ in equation (4), we get $\lambda=a-(g c /(d c+g))$, $b-(g c /(d c+g)),-c$, and $-(d g c /(d c+g))$.

The equilibrium solution $E_{8}(0,0,(d c /(d c+g))$, $(g c /(d c+g)))$ is locally asymptotically stable under the condition: $\left\{\begin{array}{l}a<(g c /(d c+g)) \\ b<(g c /(d c+g))\end{array}\right.$.
Numerical simulations agree with these results. Let $a=0.5, b=0.5, c=2, d=1, e=1, f=2$, and $g=2$. We get the stable solution $(0,0,0.5,1)$ as shown in Figure 1.

Similarly, from equilibrium point $E_{9}(0,(d b /(d b+f)), 0$, $(f b /(d b+f)))$, we can obtain the eigenvalue as follows: $\lambda=$ $a-(f b /(d b+f)), \quad c-(f b /(d b+f)),-b$, and $-(d f b /$ $(d b+f))$.

When the following conditions are met and the real part of all eigenvalues is less than 0 , it is locally asymptotically stable: $\left\{\begin{array}{l}a<(f b /(d b+f)) \\ c<(f b /(d b+f))\end{array}\right.$.

The above conclusion is proved by numerical simulation, with $a=0.5, b=2, c=0.5, d=1, e=1, f=2$, and $g=2$. The equilibrium point $(0,0.5,0,1)$ is obtained, and the simulation results are shown in Figure 2.

Similarly, from equilibrium point $E_{10}((\mathrm{~d} a /(\mathrm{d} a+e)), 0,0$, $((e a / \mathrm{d} a+e)))$, we can obtain the eigenvalue as follows: $\lambda=-a, b-(e a / d a+e), c-(e a / d a+e),-(d e a /(d a+e))$.

When the following conditions are met, it is locally asymptotically stable: $\left\{\begin{array}{l}b<e a /(d a+e) \\ c<e a /(d a+e)\end{array}\right.$.

The above conclusion is proved by numerical simulation, with $a=2, b=0.5, c=0.5, d=1, e=2, f=2$, and $g=2$. The equilibrium point $(0.5,0,0,1)$ is obtained, and the simulation results are shown in Figure 3.

By substituting the point $E 11(0,(b c d-c g+b g / b c d+c f+$ $b g),(b c d+c f-b f / b c d+c f+b g),(b c f+b c g / b c d+c f+$ $b g)$ ) in matrix (3), we can obtain the Jacobian matrix:

$$
\begin{aligned}
J & =\left[\begin{array}{cccc}
a-w(1-y z) & 0 & 0 & 0 \\
y z w & b(1-2 y)-w & 0 & -y \\
y z w & 0 & c(1-2 z)-w & -z \\
e w & f w & g w & -2 d w+f y+g z
\end{array}\right] \\
& =[a-w(1-y z)]\left[\begin{array}{ccc}
b(1-2 y)-w & 0 & -y \\
0 & c(1-2 z)-w & -z \\
f w & g w & -d w
\end{array}\right] .
\end{aligned}
$$




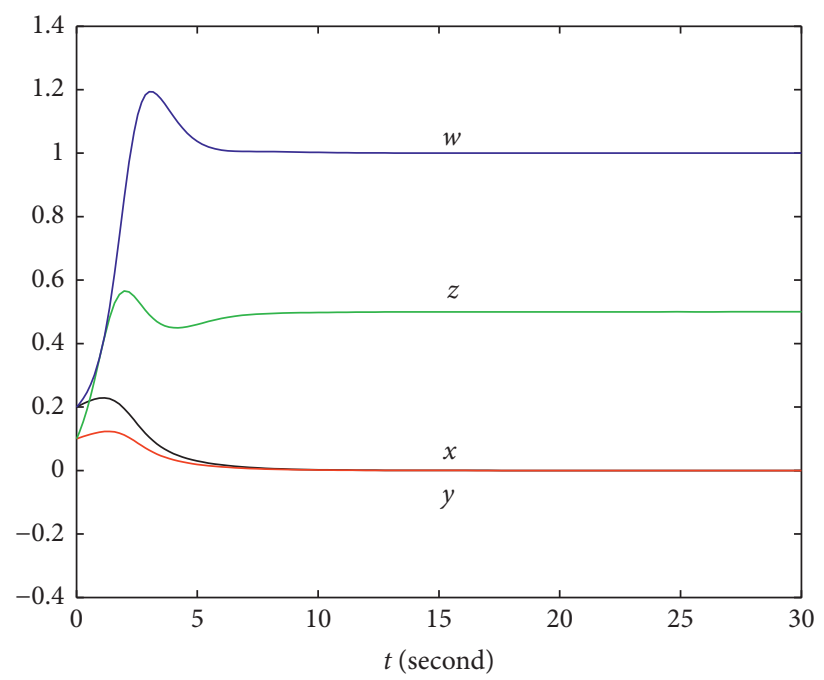

Figure 1: The values $a=0.5, b=0.5, c=2, d=1, e=1, f=2$, and $g=2$ are used. So, we get the stable solution $(x=0, y=0, z=0.5$, and $w=1)$.

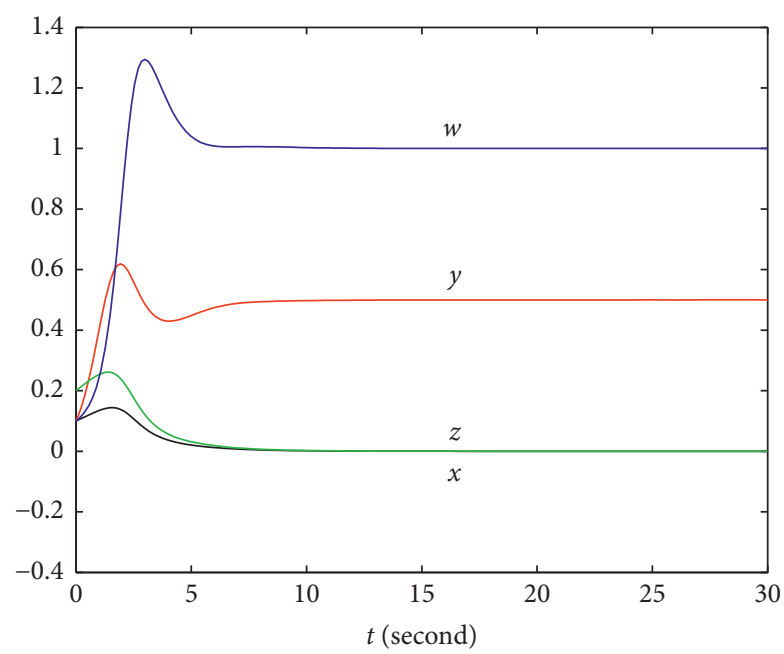

Figure 2: The values $a=0.5, b=2, c=0.5, d=1, e=1, f=2$, and $g=2$ are used. So, we get the stable solution $(x=0, y=0.5, z=0$, and $w=1)$.

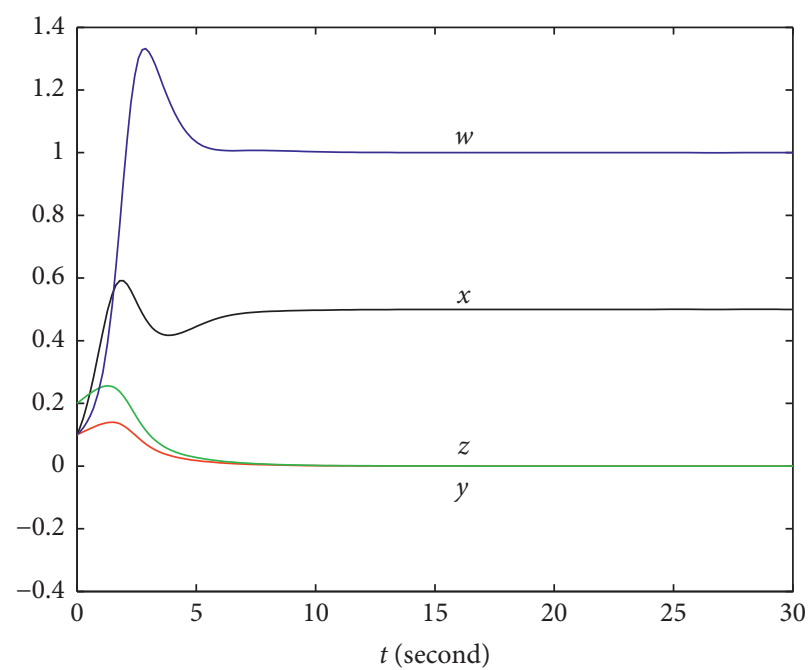

Figure 3: The values $a=2, b=0.5, c=0.5, d=1, e=2, f=2$, and $g=2$ are used. So, we get the stable solution $(x=0.5, y=0, z=0$, and $w=1)$. 
In addition, the Jacobian characteristic determinant is as follows:

$$
\begin{aligned}
|J-\lambda E| & =[a-w(1-y z)-\lambda]\left|\begin{array}{ccc}
b(1-2 y)-w-\lambda & 0 & -y \\
0 & c(1-2 z)-w-\lambda & -z \\
f w & g w & -d w-\lambda
\end{array}\right| \\
& =-(A-\lambda)\left[\lambda^{3}+(d w-B-C) \lambda^{2}+(C B+g w z+f w y-d w B-d w C) \lambda+d w B C-g w z B+f w y C\right]
\end{aligned}
$$

where $\quad A=a-w(1-y z), B=b(1-2 y)-w$, and $C=$ $c(1-2 z)-w$.

Using the Routh-Hurwitz condition, the following condition is necessary and sufficient that all roots of the characteristic equation of system (1) at the equilibrium point have a negative real part:

$$
\left\{\begin{array}{l}
A<0, \\
d w-B-C>0, \\
C B+g w z+f w y-d w B-d w C>0, \\
d w B C-g w z B+f w y C>0, \\
(d w-B-C)(C B+g w z+f w y-d w B-d w C)>d w B C-g w z B+f w y C .
\end{array}\right.
$$

The above conclusion is proved by numerical simulation, with $a=0.5, b=2, c=3, d=1, e=2, f=2$, and $g=2$. The equilibrium point $(0,0.25,0.5,1.5)$ is obtained, and the simulation results are shown in Figure 4.
Similarly, by substituting the point $E_{12}(((a c d-c g+a g) /$ $(a c d+c e+a g)), 0,((a c d+c e-a e) /(a c d+c e+a g)),((a c e+$ $a c g) /(a c d+c e+a g)))$ in matrix (3), we can obtain the Jacobian matrix:

$$
J=\left[\begin{array}{cccc}
a(1-2 x)-w & x z w & 0 & -x \\
0 & b-w(1-x z) & 0 & 0 \\
0 & x z w & c(1-2 z)-w & -z \\
e w & f w & g w & -d w
\end{array}\right]=[b-w(1-x z)]\left[\begin{array}{ccc}
a(1-2 x)-w & 0 & -x \\
0 & c(1-2 z)-w & -z \\
e w & g w & -d w
\end{array}\right]
$$

In addition, the Jacobian characteristic determinant is as follows:

$$
\begin{aligned}
|J-\lambda E| & =[b-w(1-x z)-\lambda]\left|\begin{array}{ccc}
a(1-2 x)-w-\lambda & 0 & -x \\
0 & c(1-2 z)-w-\lambda & -z \\
e w & g w & -d w-\lambda
\end{array}\right| \\
& =-(B 1-\lambda)\left[\lambda^{3}+\left(d w-A_{1}-C_{1}\right) \lambda^{2}+\left(A_{1} C_{1}+z g w+e w x-d w A_{1}-d w C_{1}\right) \lambda+d w A_{1} C_{1}-z g w A_{1}+e w x C\right]
\end{aligned}
$$

where $\left\{\begin{array}{l}A_{1}=a(1-2 x)-w \\ B_{1}=b-w(1-x z) \\ C_{1}=c(1-2 z)-w\end{array}\right.$.

Similarly, using the Routh-Hurwitz condition, the following condition is necessary and sufficient that all roots 


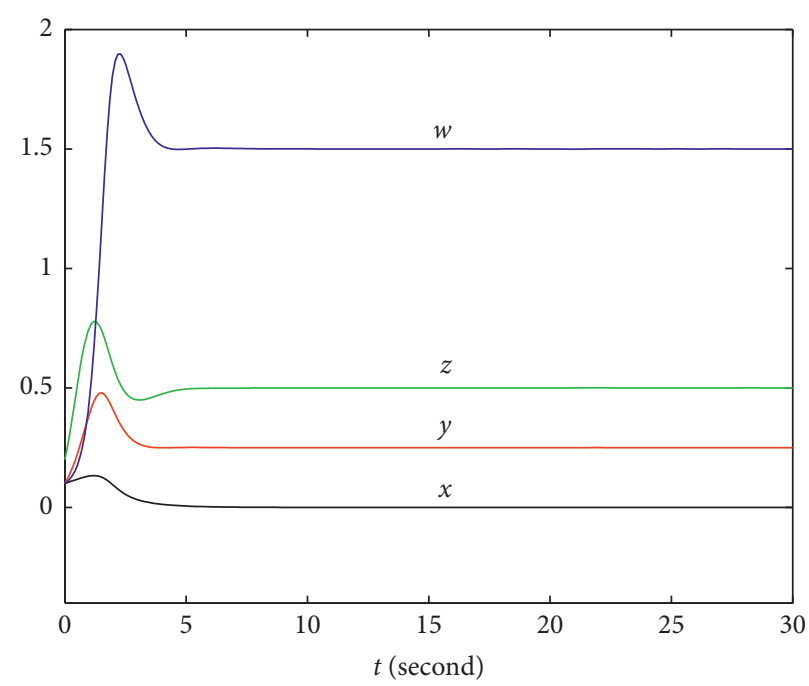

Figure 4: The values $a=0.5, b=2, c=3 ; d=1, e=2, f=2$, and $g=2$ are used. So, we get the stable solution $(x=0, y=0.25, z=0.5$, and $w=1.5)$.

of the characteristic equation of system (1) at the equi-

librium point have a negative real part:

$$
\left\{\begin{array}{l}
B_{1}<0 \\
d w-A_{1}-C_{1}>0 \\
A_{1} C_{1}+z g w+e w x-d w A_{1}-d w C_{1}>0 \\
d w A 1 C 1-z g w A_{1}+e w x C_{1}>0 \\
\left(d w-A_{1}-C_{1}\right)\left(A_{1} C_{1}+z g w+e w x-d w A_{1}-d w C_{1}\right)>d w A_{1} C_{1}-z g w A_{1}+e w x C_{1} .
\end{array}\right.
$$

The above conclusion is proved by numerical simulation, with $a=2, b=0.5, c=3, d=1, e=2, f=2$, and $g=2$. The equilibrium point $(0.25,0,0.5,1.5)$ is obtained, and the simulation results are shown in Figure 5.
By substituting the point $E_{13}(((a b d-b f+a f) /(a b d+$ $b e+a f)),((a b d+b e-a e) /(a b d+b e+a f)), 0,((a b e+a b$ $f) /(a b d+b e+a f)))$ in matrix (3), we can obtain the Jacobian matrix:

$$
J=\left[\begin{array}{cccc}
a(1-2 x)-w & 0 & x y w & -x \\
0 & b(1-2 y)-w & x y w & -y \\
0 & 0 & c-w(1-x y) & 0 \\
e w & f w & g w & -d w
\end{array}\right]=[c-w(1-x y)]\left[\begin{array}{ccc}
a(1-2 x)-w & 0 \\
0 & b(1-2 y)-w & -y \\
e w & f w & -d w
\end{array}\right]
$$

In addition, the Jacobian characteristic determinant is as follows:

$$
\begin{aligned}
|J-\lambda E| & =[c-w(1-x y)-\lambda]\left|\begin{array}{ccc}
a(1-2 x)-w-\lambda & 0 & -x \\
0 & b(1-2 y)-w-\lambda & -y \\
e w & f w & -d w-\lambda
\end{array}\right| \\
& =-(C 2-\lambda)\left[\lambda^{3}+\left(d w-A_{2}-B_{2}\right) \lambda^{2}+\left(A_{2} B_{2}+e w x+f w y-d w B_{2}-d w A_{2}\right) \lambda+d w A_{2} B_{2}-e w x B_{2}+f w y A_{2}\right]=0
\end{aligned}
$$




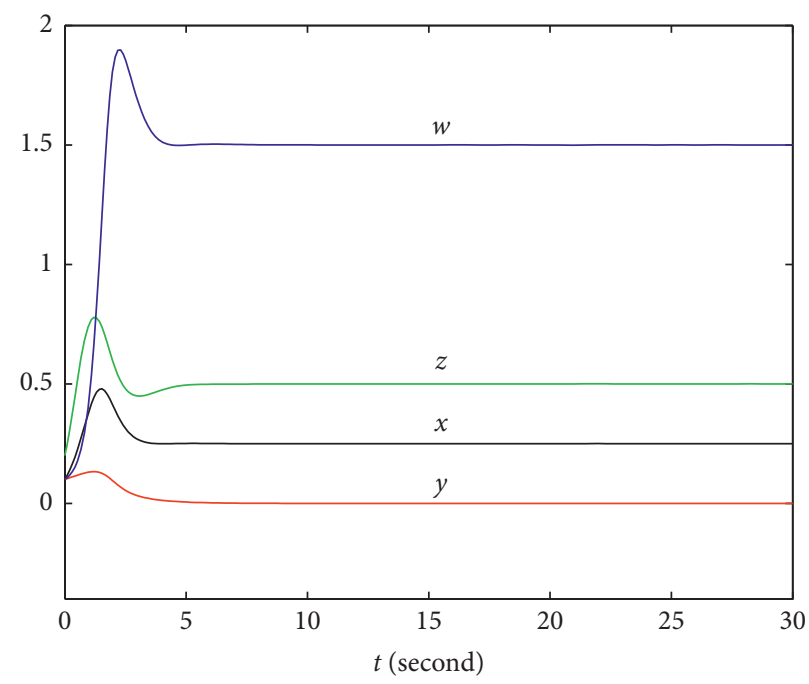

Figure 5: The values $a=2, b=0.5, c=3, d=1, e=2, f=2$, and $g=2$ are used. So, we get the stable solution $(x=0.25, y=0, z=0.5$, and $w=1.5)$.

where $\quad A_{2}=a(1-2 x)-w, B 2=b(1-2 y)-w$, and $C 2=$ $c-w(1-x y)$

Using the Routh-Hurwitz condition, the following condition is necessary and sufficient that all roots of the characteristic equation of system (1) at the equilibrium point have a negative real part:

$$
\left\{\begin{array}{l}
C_{2}<0, \\
d w-A_{2}-B_{2}>0, \\
A_{2} B_{2}+e w x+f w y-d w B_{2}-d w A_{2}>0, \\
d w A_{2} B_{2}-e w x B_{2}+f w y A_{2}>0, \\
\left(d w-B_{2}-A_{2}\right)\left(A_{2} B_{2}+e w x+f w y-d w B_{2}-d w A_{2}\right)>d w A_{2} B_{2}-e w x B_{2}+f w y A_{2} .
\end{array}\right.
$$

The above conclusion was proved by numerical simulation, with $a=2, b=3, c=0.5, d=1, e=2, f=2$, and $g=2$. The equilibrium point $(0.25,0.5,0,1.5)$ is obtained, and the simulation results are shown in Figure 6.
By substituting the point $E_{14}(1,1,1,(e+f+g) / d)$ in matrix (3), we can obtain the Jacobian matrix and the Jacobian characteristic determinant as follows:

$$
\begin{aligned}
|J-\lambda E| & =\left[\begin{array}{cccc}
-a-\lambda & w & w & 0 \\
w & -b-\lambda & w & 0 \\
w & w & -c-\lambda & 0 \\
e w & f w & g w & -e-f-g-\lambda
\end{array}\right] \\
& =(-e-f-g-\lambda)\left|\begin{array}{ccc}
-a-\lambda & w & w \\
w & -b-\lambda & w \\
w & w & -c-\lambda
\end{array}\right| \\
& =-(-e-f-g-\lambda)\left[\lambda^{3}+(a+b+c) \lambda^{2}+\left(a b+a c+b c-3 w^{2}\right) \lambda+a b c-(a+b+c) w^{2}-2 w^{3}\right]=0
\end{aligned}
$$




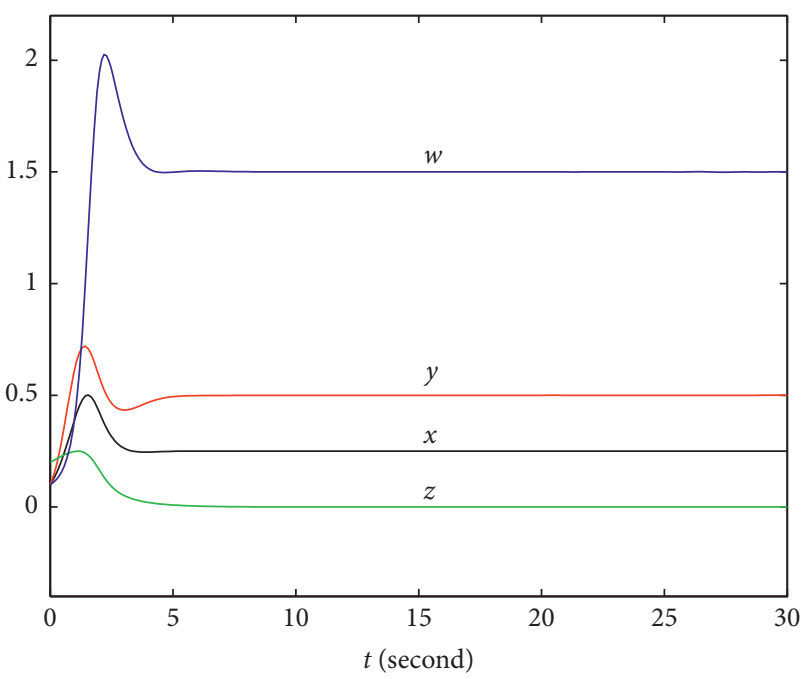

Figure 6: The values $a=2, b=3, c=0.5, d=1, e=2, f=2$, and $g=2$ are used. So, we get the stable solution $(x=0.25, y=0.5, z=0$, and $w=1.5)$.

It is known from above equation that $\lambda 1=-\mathrm{e}-\mathrm{f}-\mathrm{g}<0$. Using the Routh-Hurwitz condition, the following condition is necessary and sufficient that all roots of the characteristic equation of system (1) at the equilibrium point have a negative real part:

$$
\left\{\begin{array}{l}
a b+a c+b c-3 w^{2}>0, \\
a b c-(a+b+c) w^{2}-2 w^{3}>0, \\
a b c-(a+b+c) w^{2}-2 w^{3}<(a+b+c)\left(a b+a c+b c-3 w^{2}\right) .
\end{array}\right.
$$

When above conditions are met, $E_{14}(1,1,1,(e+$ $f+g) / d$ ) is locally asymptotically stable.

The above conclusion is proved by numerical simulation, with $a=4, b=4, c=4, d=3, e=2, f=2$, and $g=2$. The equilibrium point $(1,1,1,2)$ is obtained, and the simulation results are shown in Figure 7.

Now, we consider the above system but without team interaction help terms:

$$
\left\{\begin{array}{l}
\frac{\mathrm{d} x}{\mathrm{~d} t}=a x(1-x)-x w \\
\frac{\mathrm{d} y}{\mathrm{~d} t}=b y(1-y)-y w \\
\frac{\mathrm{d} z}{\mathrm{~d} t}=c z(1-z)-z w, \\
\frac{\mathrm{d} w}{\mathrm{~d} t}=-\mathrm{d} w^{2}+e x w+f y w+g z w .
\end{array}\right.
$$

It is easy to get its equilibrium point as follows: $x=$ $((b a g-b g c-c f b+a f c+d b c a) /(d b c a+b a g+b e c+a f c))$, $y=((d b c a+b a g+b e c-a g c-a e c) /(d b c a+b a g+b e c+a f c))$, $z=((d b c a-a f b-b e a+a f c+b e c) /(d b c a+b a g+b e c+a f c))$, and $w=(c a b(f+e+g)) /(d b c a+b a g+b e c+a f c)$.

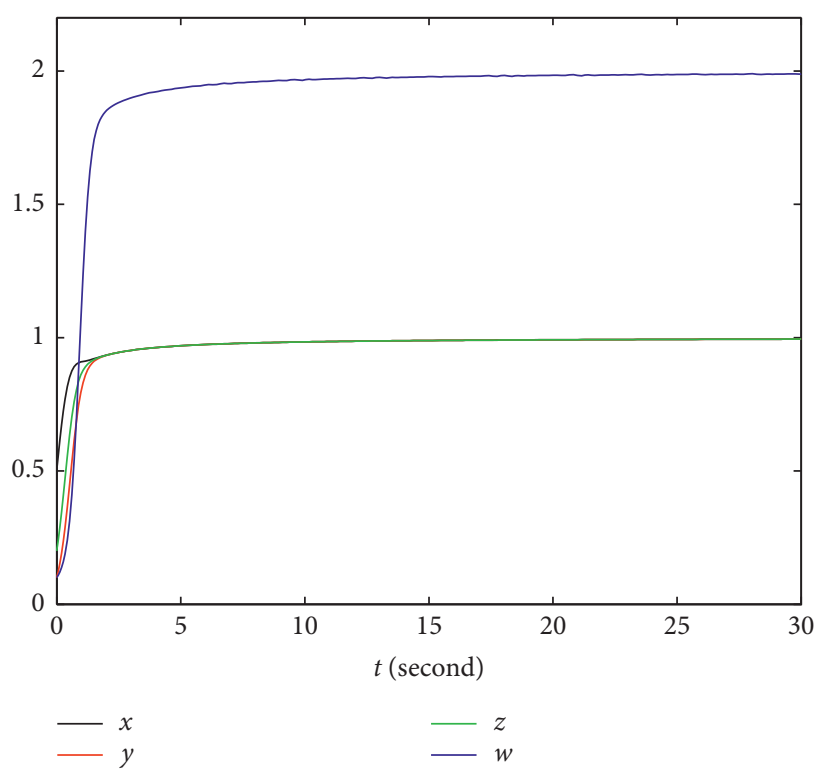

Figure 7: The values $a=4, b=4, c=4, d=3, e=2, f=2$, and $g=2$ are used. So, we get the stable solution $(x=1, y=1, z=1$, and $w=2)$.

To prove the global stability of the above equilibrium point (the internal solution) of system (16), we use the following propositions.

Proposition 2 (see [28]). The system $\mathrm{d} u / \mathrm{d} t=r u(1-u)$ has two equilibrium solutions, $u=0$ and $u=1$, where the constant $r>0$. The nonzero equilibrium solution $u=1$ is globally stable for all solutions $u$ with the initial condition $u(0)>0$.

Proposition 3. When $a, b, c, d, e, f$, and $g$ are all positive, the internal solutions of (16) are globally asymptotically stable for all solutions with the initial conditions $x(0)>0, y(0)>0$, $z(0)>0$, and $w(0)>0$.

Proof. From the first equation in system (16), it is known that $(\mathrm{d} x / \mathrm{d} t) \leq a x(1-x)$. According to Proposition 2, we can obtain that $x \leq U_{1}^{x}$, where $U_{1}^{x}=1$.

From the second equation in system (16), it is known that $(\mathrm{d} y / \mathrm{d} t) \leq b y(1-y)$. According to Proposition 2, we can obtain that $y \leq U_{1}^{y}$, where $U_{1}^{y}=1$.

From the third equation in system (16), it is known that $(\mathrm{d} z / \mathrm{d} t) \leq c z(1-z)$. According to Proposition 2, we can obtain that $z \leq U_{1}^{z}$, where $U_{1}^{z}=1$. Therefore, from the fourth equation in system (16), it is known that $(\mathrm{d} w / \mathrm{d} t) \leq-$ $\mathrm{d} w^{2}+\left(e U_{1}^{x}+f U_{1}^{y}+g U_{1}^{z}\right) w$ andd $w / \mathrm{d} t \leq \mathrm{d} w\left(\left(e U_{1}^{x}+f U_{1}^{y}+\right.\right.$ $\left.\left.g U_{1}^{z} / d\right)-w\right)$.

According to Proposition 2, we can obtain that $w \leq U_{1}^{w}$, where $U_{1}^{w}=e U_{1}^{x}+f U_{1}^{y}+g U_{1}^{z} / d$.

On the other hand,

$$
\begin{aligned}
& \frac{\mathrm{d} x}{\mathrm{~d} t} \geq \operatorname{ax}(1-x)-x U_{1}^{w}, \\
& \frac{\mathrm{d} x}{\mathrm{~d} t} \geq \operatorname{ax}\left(\left(1-\frac{U_{1}^{w}}{a}\right)-x\right) .
\end{aligned}
$$


Using Proposition 2, we can obtain that $x \geq L_{1}^{x}$, where $L_{1}^{x}=1-\left(U_{1}^{w} / a\right)$.

By the same way, $y \geq L_{1}^{y}$, where $L_{1}^{y}=1-\left(U_{1}^{w} / b\right)$, and $z \geq L_{1}^{z}$, where $L_{1}^{z}=1-\left(U_{1}^{w} / c\right)$.

Similarly, $(\mathrm{d} w / \mathrm{d} t) \geq-\mathrm{d} w^{2}+\left(e L_{1}^{x}+f L_{1}^{y}+g L_{1}^{z}\right) w \quad$ and $(\mathrm{d} w / \mathrm{d} t) \geq \mathrm{d} w\left(\left(\mathrm{e} L_{1}^{\mathrm{x}}+\mathrm{f} L_{1}^{\mathrm{y}}+\mathrm{g} L_{1}^{\mathrm{z}} / d\right)-w\right)$.

Using Proposition 2, we can obtain that $w \geq L_{1}^{w}$, where $L_{1}^{w}=\left(e L_{1}^{x}+f L_{1}^{y}+g L_{1}^{z} / d\right)$.

Continuing the above process, one gets a sequence of upper limits $U_{i}^{x}, U_{i}^{y}, U_{i}^{z}$, and $U_{i}^{w}$ and lower limits $L_{i}^{x}, L_{i}^{y}, L_{i}^{z}$, and $L_{i}^{w}$, where $i=2,3$, related by the relations:

$$
\begin{aligned}
& U_{i}^{x}=1-\frac{L_{i}^{w}}{a}, \\
& U_{i}^{y}=1-\frac{L_{i}^{w}}{b}, \\
& U_{i}^{z}=1-\frac{L_{i}^{w}}{c}, \\
& U_{i}^{w}=\frac{e U_{i}^{x}+f U_{i}^{y}+g U_{i}^{z}}{d}, \\
& L_{i}^{x}=1-\frac{U_{i}^{w}}{a}, \\
& L_{i}^{y}=1-\frac{U_{i}^{w}}{b}, \\
& L_{i}^{z}=1-\frac{U_{i}^{w}}{c}, \\
& L_{i}^{w}=\frac{e L_{i}^{x}+f L_{i}^{y}+g L_{i}^{z}}{d}, \quad i=2,3, \ldots, n,
\end{aligned}
$$

whose solutions are

$\left\{\begin{array}{l}U_{i}^{x}=L_{i}^{x}=(b a g-b g c-c f b+a f c+d b c a) /(d b c a+b a g+b e c+a f c) \\ U_{i}^{y}=L_{i}^{y}=(d b c a+b a g+b e c-a g c-a e c) /(d b c a+b a g+b e c+a f c) \\ U_{i}^{z}=L_{i}^{y}=(d b c a-a f b-b e a+a f c+b e c) /(d b c a+b a g+b e c+a f c) \\ U_{i}^{w}=L_{i}^{w}=(c a b(f+e+g)) / d b c a+b a g+b e c+a f c\end{array}\right.$.

This completes the proof.

The conclusion is proved by numerical simulation. When arbitrarily taking positive number as $a=4, b=6, c=8$, $d=2, e=3, f=2$, and $g=2$ and $x(0)=0.2, y(0)=0.1$, $z(0)=0.3$, and $w(0)=1.4$, we can obtain internal solution in equation $(16)$ as $(0.475,0.65,0.7375,2.1)$, and the simulation results are shown in Figure 8, which shows that the simulation result agrees with theory result of internal solution (equilibrium point). When arbitrarily taking positive number as $a=2, b=4, c=5, d=5, e=2, f=2$, and $g=2$ and $x(0)=1.5, y(0)=0.1, z(0)=1.3$, and $w(0)=1.4$, we can obtain internal solution in equation (16) as $(0.5652,0.7826$, $0.8261,0.8696)$, and the simulation results are shown in Figure 9, which shows that the simulation result agrees with theory result of internal solution (equilibrium point).

Proposition 4. System (16) is persistent.

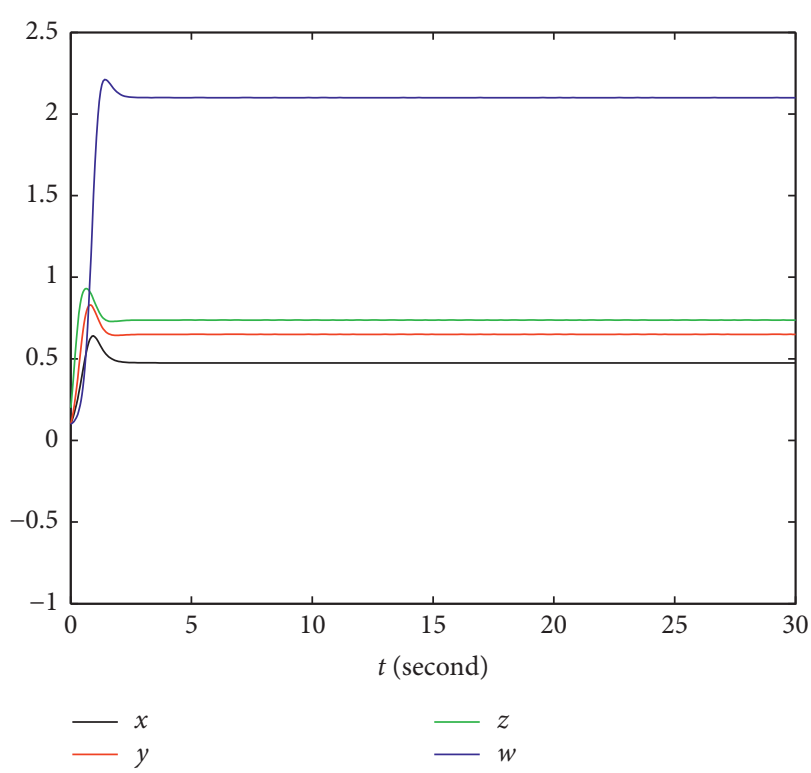

FIgURe 8: Positive parameters $a=4, b=6, c=8, d=2, e=3$, and $f=2$ corresponding to internal solution in equation $(16)$ as $(0.475,0.65$, $0.7375,2.1$

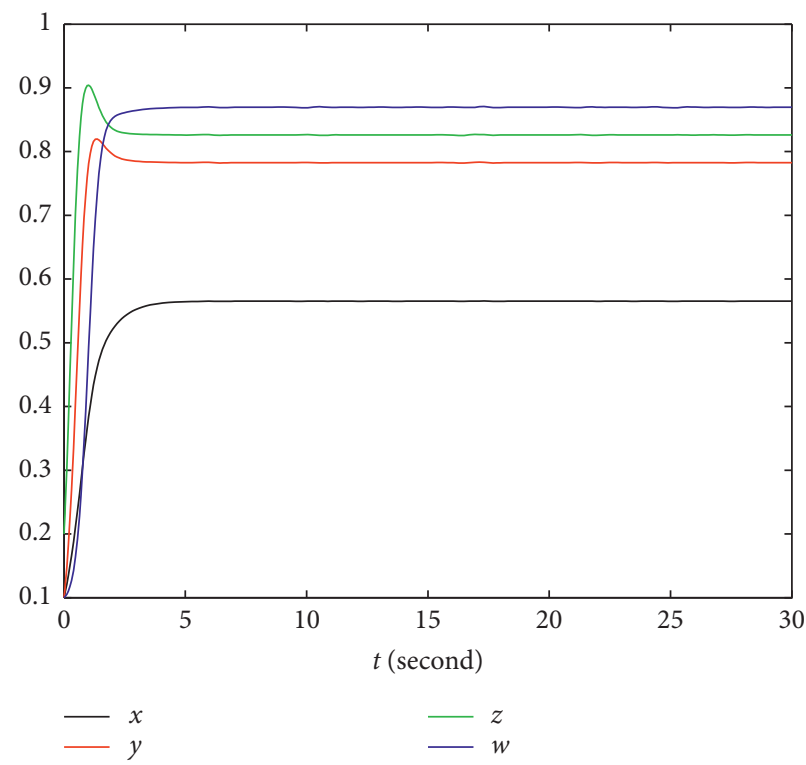

Figure 9: Positive parameters $a=2, b=4, c=5, d=5, e=2, f=2$, and $g=2$ corresponding to internal solution in equation (16) as $(0.5652,0.7826,0.8261,0.8696)$

Proof. According to Proposition 3, we have proved that for all $t>0$, all solutions with positive initial conditions satisfy $\left\{\begin{array}{l}0<L_{i}^{x} \leq x \leq U_{i}^{x} \\ L_{i}^{y} \leq y \leq U_{i}^{y} \\ L_{i}^{y} \leq z \leq U_{i}^{z} \\ L_{i}^{w} \leq w \leq U_{i}^{w}\end{array}\right.$.

Thus, system (16) is persistent. 


\section{Comparison with Reference [28]}

Reference [28] studied the two-prey one-predator model, while this paper studies the three-prey single-predator model. Compared with reference [28], our model in this paper has the following characteristics: (i) the number of differential equations for the system increases by one; (ii) the number of equilibrium points increases by half, from 8 in reference [28] to 15 in this paper; and (iii) the equilibrium point converges faster, and the convergence time of numerical simulation is about $10 \mathrm{~s}$ in reference [28] and $2 \mathrm{~s}$ in this paper.

\section{Conclusions}

In this paper, a three-prey single-predator continuous time nonlinear system is studied and a model of fourth-order nonlinear differential equation is proposed. The equilibrium point, the characteristic root, and the stability of the system solution of the differential equation are analyzed. When considering preys help each other, there are 15 equilibria in the system in which 8 equilibria are unstable and other 7 equilibria are locally asymptotically stable. When not considering preys help each other, all equilibria are globally asymptotically stable.

\section{Data Availability}

The data used to support the findings of this study are available from the corresponding author upon request.

\section{Conflicts of Interest}

The authors declare that they have no conflicts of interest.

\section{Acknowledgments}

This study was supported by the major program of the National Natural Science Foundation of China (nos. 71790593 and 71572055) and Social Science Foundation of Hunan Province of China (18YBA089).

\section{References}

[1] Q. Deng, C. Wang, and L. Yang, "Four-wing hidden attractors with one stable equilibrium point," International Journal of Bifurcation and Chaos, vol. 30, no. 6, Article ID 2050086, 2020.

[2] Q. Deng and C. Wang, "Multi-scroll hidden attractors with two stable equilibrium points," Chaos: An Interdisciplinary Journal of Nonlinear Science, vol. 29, no. 9, Article ID 093112, 2019.

[3] X. Zhang, C. Wang, W. Yao, and H. Lin, "Chaotic system with bondorbital attractors," Nonlinear Dynamics, vol. 97, no. 4, pp. 2159-2174, 2019.

[4] C. Li, W. Joo-Chen Thio, H. T. Lu, and L. Tian, "A memristive chaotic oscillator with increasing amplitude and frequency," IEEE Access, vol. 6, pp. 12945-12950, 2018.

[5] C. Li, W. Joo-Chen Thio, J. C. Sprott, H. H.-C. Iu, and Y. Xu, "Constructing infinitely many attractors in a programmable chaotic circuit,” IEEE Access, vol. 6, pp. 29003-29012, 2018.
[6] Q. Zhao, C. Wang, and X. Zhang, "A universal emulator for memristor, memcapacitor, and meminductor and its chaotic circuit," Chaos, vol. 29, no. 1, Article ID 013141, 2019.

[7] C. Wang, H. Xia, and L. Zhou, "A memristive hyperchaotic multiscroll Jerk system with controllable scroll numbers," International Journal of Bifurcation and Chaos, vol. 27, no. 6, Article ID 1750091, 2017.

[8] R. Wu and C. Wang, "A new simple chaotic circuit based on memristor," International Journal of Bifurcation and Chaos, vol. 26, no. 9, Article ID 1650145, 2016.

[9] H. Lin, C. Wang, Y. Tan, and Y. M. Tan, "Hidden extreme multistability with hyperchaos and transient chaos in a hopfield neural network affected by electromagnetic radiation," Nonlinear Dynamics, vol. 99, no. 3, pp. 2369-2386, 2020.

[10] W. Yao, C. Wang, J. Cao, Y. Sun, and C. Zhou, "Hybrid multisynchronization of coupled multistable memristive neural networks with time delays," Neurocomputing, vol. 363, pp. 281-294, 2019.

[11] C. Zhou, C. H. Wang, Y. C. Sun, and W. Yao, "Weighted sum synchronization of memristive coupled neural networks," Neurocomputing, vol. 403, pp. 211-223, 2020.

[12] W. Yao, C. Wang, Y. Sun, C. Zhou, and H. Lin, "Synchronization of inertial memristive neural networks with timevarying delays via static or dynamic event-triggered control," Neurocomputing, vol. 404, pp. 367-380, 2020.

[13] H. R. Lin, C. H. Wang, W. Yao, and Y. M. Tan, "Chaotic dynamics in a neural network with different types of external stimuli," Communications in Nonlinear Science and Numerical Simulation, vol. 90, Article ID 105390, 2020.

[14] C. Wang, L. Xiong, J. Sun, and W. Yao, "Memristor-based neural networks with weight simultaneous perturbation training," Nonlinear Dynamics, vol. 95, no. 4, pp. 2893-2906, 2019.

[15] Y. M. Tan and C. H. Wang, "A simple locally active memristor and its application in HR neurons," Chaos: An Interdisciplinary Journal of Nonlinear Science, vol. 30, no. 5, Article ID 053118, 2020.

[16] S. C. Wang, C. H. Wang, and C. Xu, "An image encryption algorithm based on a hidden attractor chaos system and the Knuth-Durstenfeld algorithm," Optics and Lasers in Engineering, vol. 128, Article ID 105995, 2020.

[17] C. Xu, J. R. Sun, and C. H. Wang, "An image encryption algorithm based on random walk and hyperchaotic systems," International Journal of Bifurcation and Chaos, vol. 30, no. 4, Article ID 2050060, 2020.

[18] M. J. Zhou and C. H. Wang, "A novel image encryption scheme based on conservative hyperchaotic system and closed-loop diffusion between blocks," Signal Processing, vol. 171, Article ID 107484, 2020.

[19] M. L. Rosenzweig and R. H. MacArthur, "Graphical representation and stability conditions of predator-prey interactions," The American Naturalist, vol. 97, no. 895, pp. 209-223, 1963.

[20] S.-B. Hsu and T.-W. Huang, "Global stability for a class of predator-prey systems," SIAM Journal on Applied Mathematics, vol. 55, no. 3, pp. 763-783, 1995.

[21] H. I. Freedman, "Stability analysis of a predator-prey system with mutual interference and density-dependent death rates," Bulletin of Mathematical Biology, vol. 41, no. 1, pp. 67-78, 1979.

[22] Y. Q. Wang and Z. J. Jing, "Multiple limit cycles and global stability in predator-prey model," ACTA Mathematicae Applicatae Sinica, vol. 15, no. 2, pp. 206-219, 1999.

[23] Z. Zhang and Z. Hou, "Existence of four positive periodic solutions for a ratio-dependent predator-prey system with 
multiple exploited (or harvesting) terms," Nonlinear Analysis: Real World Applications, vol. 11, no. 3, pp. 1560-1571, 2010.

[24] J. Llibre and D. Xiao, "Global dynamics of a Lotka--volterra model with two predators competing for one prey," SIAM Journal on Applied Mathematics, vol. 74, no. 2, pp. 434-453, 2014.

[25] Y. Wang, H. Wu, and D. L. DeAngelis, "Global dynamics of a mutualism-competition model with one resource and multiple consumers," Journal of Mathematical Biology, vol. 78, no. 3, pp. 683-710, 2019.

[26] Y. Wang and H. Wu, "Global dynamics of Lotka-Volterra equations characterizing multiple predators competing for one prey," Journal of Mathematical Analysis and Applications, vol. 491, no. 1, Article ID 124293, 2020.

[27] M. F. Elettreby and H. El-Metwally, "Multi-team preypredator model," International Journal of Modern Physics C, vol. 18, no. 10, pp. 1609-1617, 2007.

[28] M. F. Elettreby, "Two-prey one-predator model," Chaos, Solitons \& Fractals, vol. 39, no. 5, pp. 2018-2027, 2009. 